\title{
Pancreatic Origin Hepatoid Adenocarcinoma with Liver Metastasis
}

\section{Salehi M ${ }^{1}$, Nwachukwu N¹, Grijalva $V^{1}$, Li LZ $^{1}$ and} Weinstein PL ${ }^{2 *}$

${ }^{1}$ Department of Internal Medicine, Stamford Hospital, USA

${ }^{2}$ Department of Hematology and Oncology, Stamford Hospital, USA

*Corresponding author: Weinstein PL, Department of Hematology and Oncology, Stamford Hospital, One Hospital Plaza, P.O. Box 9317, Stamford, CT 06904, USA

Received: July 08, 2021; Accepted: July 24, 2021;

Published: July 31, 2021

\begin{abstract}
Hepatoid Adenocarcinoma (HAC) is a rare form of aggressive extrahepatic neoplasm with similar morphologic features to Hepatocellular Carcinoma $(\mathrm{HCC})$. HAC with pancreatic origin is rare and the exact incidence is unknown. Given shared morphological and Immunohistochemical (IHC) characteristics, it may be difficult to distinguish metastatic HAC with liver involvement from primary HCC. We present a rare case of ductal type pancreatic hepatoid adenocarcinoma involving the pancreatic head, ampulla of Vater, and liver, and illustrate strategies for diagnosis and treatment.
\end{abstract}

A 65-year-old woman presented with epigastric pain, vomiting, melena, and weight loss. There was direct hyperbilirubinemia with elevated hepatic markers. Imaging displayed a pancreatic head mass with moderate biliary obstruction and a hepatic lobe lesion. Fine needle biopsy of the liver mass initially was consistent with $\mathrm{HCC}$, and biopsies of the pancreatic mass and ampulla walls showed pancreatic adenocarcinoma. Due to the unusual finding of co-existing HCC and pancreatic adenocarcinoma, the hepatic mass biopsy was sent for external evaluation, which revealed poorly differentiated adenocarcinoma, consistent with $\mathrm{HAC}$ of pancreatic origin. The tumor was positive for mucicarmine stain, HepPar1, CEA, and CK7. It was negative for MOC-31, Arginase, ALBISH, MGB, ER, TTF-1, GCDFP15, CK-20 and CDX2, thus confirming HAC. The patient was referred to outpatient chemotherapy with gemcitabine and paclitaxel however demonstrated progression and expired following recurrent bilateral pulmonary emboli.

HAC may present with non-specific symptoms, is highly aggressive, and may be difficult to distinguish from primary HCC from histopathologic characteristics alone. Accurate diagnosis requires clinicohistopathologic and IHC analysis.

Keywords: Hepatoid adenocarcinoma; Liver metastasis; Tumor markers; Hepatocellular carcinoma; Immunohistochemistry

\section{Abbreviations}

HAC: Hepatoid Adenocarcinoma; HCC: Hepatocellular Carcinoma; GI: Gastrointestinal; GU: Genitourinary; IHC: Immunohistochemical; RUQ: Right UpperQuadrant;LUQ: Left Upper Quadrant; AFP: $\alpha$-Fetoprotein; AST: Aspartate Aminotransferase; ALT: Alanine Aminotransferase; ALP: Alkaline Phosphatase; GGT: Gamma-Glutamyltransferase; CT: Computed Tomography; MRCP: Magnetic Resonance Cholangiopancreatography; CA199: Carbohydrate Antigen 19-9; CEA: Carcinoembryonic Antigen; HepPar1: Anti-Hepatocyte Specific Antigen; CK: Cytokeratin; MOC-31: Epithelial Specific Antigen; ISH: In-Situ Hybridization; MGB: Mammaglobin; ER: Estrogen Receptor; TTF-1: Thyroid Transcription Factor 1; GCDFP15: Gross Cystic Disease Fluid Protein 15; CDX2: Caudal Type Homeobox 2; PET: Positron Emission Tomography; IVC: Inferior Vena Cava; PHAC: Pancreatic Hepatoid Adenocarcinoma; PIVKA-II: Protein Induced by Vitamin K Absence or Antagonist-II; DVT: Deep Vein Thrombosis; PE: Pulmonary Embolism

\section{Introduction}

Hepatoid Adenocarcinoma (HAC) is a rare form of extrahepatic neoplasm with morphologic features similar to Hepatocellular Carcinoma (HCC) and with hepatocyte differentiation [1,2]. The most common site of origin is the stomach, presumably because both the stomach and liver share the same embryonic origin from the foregut [3-5]. HAC may also rarely involve the lungs, peritoneum, omentum, areas of the Gastrointestinal (GI) and Genitourinary (GU) tracts as well [6-11].

HAC with pancreatic origin is extremely rare and the exact incidence is unknown. Owing to its rarity, its clinical course and features are still not fully understood [12-14]. It may also be metastatic at presentation, commonly affecting the liver, lymph nodes, and lungs [15].

HCC often shares similar serological, morphological and Immunohistochemical (IHC) characteristics with HAC. Shared laboratory findings include elevated $\alpha$-fetoprotein (AFP), HepPar-1, glypican-3, arginase, and albumin [16]. Thus, it is difficult to distinguish metastatic HAC with liver involvement from primary HCC.

We present a rare case of a female with ductal type pancreatic hepatoid adenocarcinoma in the head and ampulla of Vater with liver 
involvement.

\section{Case Presentation}

A 65-year-old female nonsmoker, without history of alcohol use was referred to the emergency department from her primary care provider with weakness, epigastric pain, nausea, vomiting for a month. There was unintentional weight loss of 25 pounds over three months and tarry black stools which subsequently turned light in color. Physical exam was remarkable for jaundice, and epigastric tenderness to palpation.

Laboratory studies demonstrated total bilirubin of $3.7 \mathrm{mg} / \mathrm{dl}$, direct bilirubin $2.6 \mathrm{mg} / \mathrm{dl}$, AST $239 \mathrm{U} / \mathrm{L}$, ALT $316 \mathrm{U} / \mathrm{L}$, ALP $1073 \mathrm{U} / \mathrm{L}$ and GGT 665 U/L. Hepatitis panel was negative. Initial ultrasound demonstrated heterogenous liver with left hepatic lobe cyst, and biliary ductal dilation measuring $2.0 \mathrm{~cm}$. Non contrast CT scan of the abdomen and pelvis was initially unremarkable, subsequent MRCP revealed a $3 \mathrm{~cm}$ hypoenhancing pancreatic head mass with moderate biliary obstruction and redemonstrated left hepatic lesion. There was thickening of the third portion of the duodenum and also a $2 \mathrm{~cm}$ lesion in the anterior lateral segment of the left hepatic lobe. Initial tumor markers revealed a normal AFP of $2.6 \mathrm{ng} / \mathrm{ml}$ and elevated CA19-9 of $1879 \mathrm{U} / \mathrm{ml}$, and CEA of $33.2 \mathrm{ng} / \mathrm{ml}$. She was treated with stenting for biliary obstruction. Fine needle biopsy of the liver mass initially was consistent with HCC. Endoscopic ultrasound redemonstrated a 5.0 x $3.5 \mathrm{~cm}$ mass, involving the pancreatic head and uncinate process with encasement of superior mesenteric veins and ampulla wall. Biopsies of the pancreatic mass and ampulla walls showed pancreatic adenocarcinoma.

Due to the rarity of co-existing primary HCC and pancreatic adenocarcinoma, the hepatic mass biopsy was sent for external evaluation, which revealed poorly differentiated adenocarcinoma, consistent with $\mathrm{HAC}$ of pancreatic origin. The tumor cells were positive for mucicarmine stain, HepPar1, CEA, and CK7. It was negative for MOC-31, Arginase, ALB-ISH, MGB, ER, TTF-1, GCDFP15, CK-20 and CDX2, thus confirming HAC.

The patient was referred to outpatient chemotherapy with gemcitabine and protein-bound paclitaxel (Abraxane), and with subsequent reduction in CA 19-9 levels. CT scan of the abdomen two months after treatment initiation revealed increased size of multiple mesenteric nodes with new abdominal wall metastasis. PET scan showed hypermetabolic liver metastasis and lymphadenopathy of the retroperitoneum. Gemcitabine and paclitaxel were suspended for two months due to weakness, fatigue, and hospitalization for pulmonary embolus. during hospitalization for pulmonary embolus. Subsequently she was restarted on gemcitabine and paclitaxel however required frequent suspension of treatment due to recurrent abdominal pain and recurrent DVT/PE. The patient ultimately expired six months after diagnosis, following hospitalization for recurrent bilateral pulmonary emboli, and malignant hypercoagulation.

\section{Discussion}

Ductal pancreatic adenocarcinoma is the most common type of pancreatic malignancy, with hepatoid adenocarcinoma being categorized as one of its rare subdivisions [17]. The term Hepatoid adenocarcinoma comes from "liver-like" tumor, which resembles HCC. This rare neoplasm was first proposed by Ishikura et al. in 1985

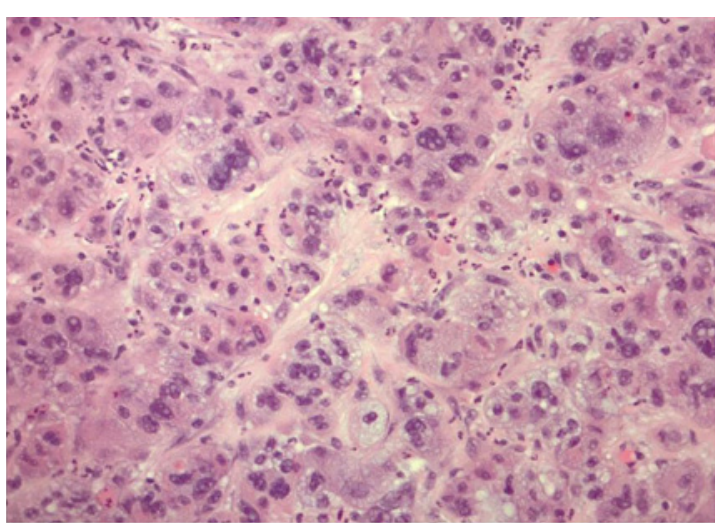

Figure 1: Section of liver, Hematoxylin and Eosin stain, 200x magnification light microscopy.

Nets and trabecules of polyglonal cells with nuclear pleomorphism and abundant eosinophilic cytoplasm.

who identified an AFP producing gastric tumor with IHC features of hepatocytes [18]. In 1987, the first case with pancreatic origin was described [19]. Since then, only a few cases of Pancreatic HAC (PHAC) have been reported and the exact features and behavior of this tumor remain unclear. A recent review published March 2020, reported a total of 39 hepatoid carcinoma cases with pancreatic origins [14]. In our English literature search May 2021, no other PHAC has been described since.

Histologically, PHAC can be divided in two categories; either pure, or a combined type which may contain features of acinar, ductal cell, mucin, neuroendocrine, islet cell glucagonoma, and serous microcystic adenoma. Combined types are less common, have a higher recurrence rate, and worse survival [14,20,21]. Zeng SX et al. study identified only 3 cases with ductal component. We reported the 4th ductal type HAC of pancreas, located in the head and extended to the ampulla. Head of the pancreas lesions have been listed as the second most common site of tumor origin after the tail. Furthermore, PHAC is more common in males; females account for $32.5 \%$ of reported cases including ours [14]. The liver is the second most metastasis site after lymph nodes, $46.3 \%$ and $57.5 \%$ respectively [9]. Per Zeng SX et al. among all pancreatic carcinomas, $43.58 \%$ of have metastasized. Among those, liver involvement was present in 12 out of 17 cases (70.5\%) [14]. Usually metastasis is found at the time of presentation but there was a report that after PHAC was removed, liver involvement developed almost a year later [22].

The present case also metastasized to the liver, but initially it was reported as HCC. IHC evaluation is critical given the similar morphologies between HAC and HCC. This patient with both pancreas and liver masses had a biopsy result consistent with adenocarcinoma of pancreas and HCC. It is very rare to have synchronous primary liver and pancreas cancers, and only three cases have been reported in the literature [23]. Furthermore, normal AFP, no evidence of cirrhosis or hemochromatosis, negative hepatitis panel, no consumption of alcohol and negative Arginase-I are very unlikely in HCC [24]. Given the above concerns, a second review of the biopsy at the Mayo Clinic revealed the final diagnosis.

The diagnosis of hepatoid carcinoma is mainly histologic although there are no established criteria. Specific pathological 
features are medium to large polygonal cells with abundant glycogen, bile production, and features of eosinophilia, granularity and hyaline globules within the cytoplasm. These cells can appear in trabecular, medullary or glandular patterns. Similarity to hepatocytes may make HAC indistinguishable from HCC, requiring IHC stains to differentiate the two [25-29].

Different IHC markers have been reported to be useful in diagnosis such as CK, AFP, Hep-Par 1, glypican-3, arginase, albumin, Sall-4, CEA, CD10, and PIVKA-II; these can also be positive in HCC and thus are not specific $[9,16,22,30]$. Arginase- 1 is usually positive only in HCC and could help in differentiating HCC and HAC [31]. CK-19 positivity is also mentioned as a distinguishing marker for HAC [15]. AFP is normally produced by the fetal liver and yolk sac has almost $86 \%$ specificity for early HCC detection [32]. Elevated AFP has been described as a hepatocyte differentiation marker for hepatoid carcinomas [33]. However, in PHAC, AFP was expressed only in $57 \%$ of cases and other hepatocyte markers were used instead to support the diagnosis [16]. In one study of 408 HAC cases with different origins by Sun et al. HepParl was also deemed to be a specific marker for HAC; being positive in all tested pancreatic origin cases.

In our case, the liver biopsy sample was not tested for CK-19, but was positive for CK7 which supports the diagnosis of adenocarcinoma [24]. It was positive for Hep-Par 1, CEA, and negative for Arginase, supporting HAC.

Prior studies recommended resection of tumor as a first treatment option if possible, with adjuvant chemotherapy [34]. However, in our case, as a metastatic disease and location of tumor, made her inoperable. Palliative chemotherapy regimens have been described for unresectable HAC cases, as well as adjuvant and neoadjuvant options with different survival benefits including 5-FU, Adriamycin, Gemcitabine, Cisplatin, Irinotecan, Carboplatin, Sorafenib, and modified-FOLFIRINOX $[15,35,36]$. Our patient was not a fit candidate for modified-FOLFIRINOX and thus was offered Gemcitabine and Abraxane. In almost one month, patient CA19-9 fell to 1088 from 2438 (repeated prior to starting the treatment). During treatment she had recurring bouts of abdominal pain, often requiring hospitalization and treatment interruption. She additionally developed multiple DVTs and PEs. This same complication happened to one of the HPAC cases reported by Yang et al occurred one-month post PHAC resection without identifiable metastasis [37]. Thrombogenicity is not an uncommon feature of pancreatic neoplasms [38].

Unfortunately, the prognosis of PHAC remains poor. Late stage on presentation, rarity of the disease with no established standard treatment, and the aggressive nature of the tumor all contribute to the unfavorable prognosis [30,36,39].

\section{References}

1. Simmet $V$, Noblecourt $M$, Lizée $T$, Morvant $B$, Girault $S$, Soulié $P$, et al Chemotherapy of metastatic hepatoid adenocarcinoma: Literature review and two case reports with cisplatin etoposide. Oncol Lett. 2018; 15: 48-54.

2. Metzgeroth G, Ströbel P, Baumbusch T, Reiter A, Hastka J. Hepatoid adenocarcinoma - review of the literature illustrated by a rare case originating in the peritoneal cavity. Onkologie. 2010; 33: 263-269.

3. Zeng X, Zhang P, Xiao H, Wu X, Liu W, He J, et al. Clinicopathological features and prognosis of intestinal hepatoid adenocarcinoma: evaluation of a pooled case series. Oncotarget. 2018; 9: 2715-2725.
4. Yang J, Wang R, Zhang W, Zhuang W, Wang M, Tang C. Clinicopathological and prognostic characteristics of hepatoid adenocarcinoma of the stomach. Gastroenterol Res Pract. 2014; 2014: 140587.

5. Kashani A, Ellis JC, Kahn M, Jamil LH. Liver metastasis from hepatoid adenocarcinoma of the esophagus mimicking hepatocellular carcinoma. Gastroenterol Rep (Oxf). 2017; 5: 67-71.

6. Veerankutty FH, Yeldho V, Tu SA, Venugopal B, Manoj KS, Vidhya C. Hepatoid carcinoma of the pancreas combined with serous cystadenoma: a case report and review of the literature. Hepatobiliary Surg Nutr. 2015; 4: 354-362.

7. Paner GP, Thompson KS, Reyes CV. Hepatoid carcinoma of the pancreas. Cancer. 2000; 88: 1582-1589.

8. Haninger DM, Kloecker GH, Bousamra li M, Nowacki MR, Slone SP. Hepatoid adenocarcinoma of the lung: report of five cases and review of the literature. Mod Pathol. 2014; 27: 535-542.

9. Su JS, Chen YT, Wang RC, Wu CY, Lee SW, Lee TY. Clinicopathological characteristics in the differential diagnosis of hepatoid adenocarcinoma: a literature review. World J Gastroenterol. 2013; 19: 321-327.

10. Kostakopoulos NA, Karakousis ND, Linardoutsos D, Argyropoulos V, Kouroupakis P, Kastora S, et al. A Giant Hepatoid Carcinoma of the Perirenal Fat With Very High A-Fetoprotein and Vitamin B12 Levels. Am Surg. 2020: 3134820952824

11. Zou M, Li Y, Dai Y, Sun L, Huang T, Yuan X, et al. AFP-producing hepatoid adenocarcinoma (HAC) of peritoneum and omentum: a case report and literature review. OncoTargets and therapy. 2019; 12: 7649.

12. Kuo PC, Chen SC, Shyr YM, Kuo YJ, Lee RC, Wang SE. Hepatoid carcinoma of the pancreas. World J Surg Oncol. 2015; 13: 185.

13. Williams NL, Palmer JD, Bar-Ad V, Anné PR, Sama AR, Weinstein JC, et al. Hepatoid Carcinoma of the Pancreas: A Case Report and Review of the Literature. Case Rep Pancreat Cancer. 2015; 1: 3-6.

14. Zeng S-X, Tan S-W, Fong C-JTH, Liang Q, Zhao B-L, Liu K, et al. Hepatoid carcinoma of the pancreas: A case report and review of the literature. World J Clin Cases. 2020; 8: 1116-1128.

15. Petrelli F, Ghilardi M, Colombo S, Stringhi E, Barbara C, Cabiddu M, et al. A rare case of metastatic pancreatic hepatoid carcinoma treated with sorafenib. J Gastrointest Cancer. 2012; 43: 97-102.

16. Sun B, Jain R, Bhattacharyya A, Cunningham J. Diagnosis of Hepatoid Carcinoma of Extrahepatic Origins-Cell Markers and Pathologic Standards. Biomedical Journal of Scientific \& Technical Research. 2019; 15: 11182 11187.

17. Haeberle L, Esposito I. Pathology of pancreatic cancer. Translational gastroenterology and hepatology. 2019; 4.

18. Ishikura H, Fukasawa Y, Ogasawara K, Natori T, Tsukada Y, Aizawa M. An AFP-producing gastric carcinoma with features of hepatic differentiation. A case report. Cancer. 1985; 56: 840-848.

19. Hruban RH, Molina JM, Reddy MN, Boitnott JK. A neoplasm with pancreatic and hepatocellular differentiation presenting with subcutaneous fat necrosis. Am J Clin Pathol. 1987; 88: 639-645.

20. Chang JM, Katariya NN, Lam-Himlin DM, Haakinson DJ, Ramanathan RK, Halfdanarson TR, et al. Hepatoid Carcinoma of the Pancreas: Case Report, Next-Generation Tumor Profiling, and Literature Review. Case Reports in Gastroenterology. 2016; 10: 605-612.

21. Tomino T, Ninomiya M, Matono R, Narutomi F, Oshiro $Y$, Watanabe $K$, et al. Pure pancreatic hepatoid carcinoma: a surgical case report and literature review. Surgical case reports. 2019; 5: 1-7.

22. Matsueda K, Yamamoto H, Yoshida Y, Notohara K. Hepatoid carcinoma of the pancreas producing protein induced by vitamin $\mathrm{K}$ absence or antagonist II (PIVKA-II) and $\alpha$-fetoprotein (AFP). Journal of Gastroenterology. 2006; 41: 1011-1019.

23. Pan P-Y, Lai P-S, Hsiao C-Y. A Patient with Synchronous Primary Hepatocellular Carcinoma and Primary Pancreatic Ductal Adenocarcinoma. 
Indian Journal of Surgery. 2019; 81: 596-598.

24. Qian X, Zhou D, Gao B, Wang W. An alpha-fetoprotein-negative hepatoid adenocarcinoma of the gallbladder with squamous differentiation. Hepatobiliary Surg Nutr. 2020; 9: 116-118.

25. Hameed O, Xu H, Saddeghi S, Maluf H. Hepatoid carcinoma of the pancreas: a case report and literature review of a heterogeneous group of tumors. The American journal of surgical pathology. 2007; 31: 146-152.

26. Jung JY, Kim YJ, Kim HM, Kim HJ, Park SW, Song SY, et al. Hepatoid carcinoma of the pancreas combined with neuroendocrine carcinoma. Gut and liver. 2010; 4: 98

27. Xin B-B, Li J-A, Han X, Zhao J, Ji Y, Lou W-H, et al. Successful treatment of a case with pancreatic neuroendocrine carcinoma with focal hepatoid differentiation: a case report and literature review. International journal of clinical and experimental medicine. 2014; 7: 3588.

28. Kai K, Nakamura J, Ide T, Masuda M, Kitahara K, Miyoshi A, et al. Hepatoid carcinoma of the pancreas penetrating into the gastric cavity: a case report and literature review. Pathology international. 2012; 62: 485-90.

29. Tong L, Pan H, He J, Weng M, Zheng L. Hepatoid adenocarcinoma arising from heterotopic pancreas of the ileum: a case report. Medicine. 2016; 95.

30. Ferreira BP, Vasquez J, Carilli A. Metastatic hepatoid carcinoma of the pancreas: first description of treatment with capecitabine and temozolomide. The American journal of the medical sciences. 2017;353(6):610-2

31. Miyama Y, Fujii T, Murase K, Takaya H, Kondo F. Hepatoid adenocarcinoma of the lung mimicking metastatic hepatocellular carcinoma. Autopsy and Case Reports. 2020; 10.

32. Lok AS, Sterling RK, Everhart JE, Wright EC, Hoefs JC, Di Bisceglie AM, et al. Des-gamma-carboxy prothrombin and alpha-fetoprotein as biomarkers for the early detection of hepatocellular carcinoma. Gastroenterology. 2010; 138: 493-502.
33. Hocking GR, Shembrey M, Hay D, Östör AG. Alpha-fetoprotein-producing adenocarcinoma of the sigmoid colon with possible hepatoid differentiation. Pathology. 1995; 27: 277-279.

34. Chaudhari R, Murphy K, Schwartz S, Chaudhari J, Ho I, Nunes F. Hepatoid Adenocarcinoma Presenting as Pancreatitis. ACG Case Reports Journal. 2020; 7 .

35. Vanoli A, Argenti F, Vinci A, La Rosa S, Viglio A, Riboni R, et al. Hepatoid carcinoma of the pancreas with lymphoid stroma: first description of the clinical, morphological, immunohistochemical, and molecular characteristics of an unusual pancreatic carcinoma. Virchows Archiv. 2015; 467: 237-245.

36. Ma T, Bai X, Li G, Wei S, Liang T. Neoadjuvant modified-FOLFIRINOX followed by surgical resection of both the primary and metastatic tumors of a pancreatic hepatoid carcinoma with synchronous liver metastasis: A case report. Medicine. 2017; 96.

37. Yang C, Sun L, Lai JZ, Zhou L, Liu Z, Xi Y, et al. Primary Hepatoid Carcinoma of the Pancreas: A Clinicopathological Study of 3 Cases With Review of Additional 31 Cases in the Literature. International Journal of Surgical Pathology. 2018; 27: 28-42.

38. Khorana AA, Dalal M, Lin J, Connolly GC. Incidence and predictors of venous Thromboembolism (VTE) among ambulatory high-risk cancer patients undergoing chemotherapy in the United States. Cancer. 2013; 119: 648-655.

39. Veerankutty FH, Yeldho V, Shabeer Ali T, Venugopal B, Manoj KS, Vidhya C. Hepatoid carcinoma of the pancreas combined with serous cystadenoma: a case report and review of the literature. Hepatobiliary surgery and nutrition. 2015; 4: 354 